\title{
Cistanche tubulosa induces reactive oxygen species-mediated apoptosis of primary and metastatic human colon cancer cells
}

\author{
Afnan Saleh Al-Menhali ${ }^{1}$, Safya Ali Jameela ${ }^{1}$, Aishah A. Latiff ${ }^{1}$, Mohamed A. Elrayess ${ }^{2}$, Mohammed Alsayrafi ${ }^{1}$, \\ Morana Jaganjac ${ }^{1} *$ \\ ${ }^{1}$ Anti Doping Lab Qatar, Toxicology and Multipurpose, Doha, Qatar. \\ ${ }^{2}$ Anti Doping Lab Qatar, Life Science Research, Doha, Qatar.
}

\section{ARTICLE INFO}

Article history:

Received on: 01/11/2016

Accepted on: 07/01/2017

Available online: 30/05/2017

Key words:

Cistanche tubulosa, colon

cancer, redox homeostasis,

anticancer bioactivity.

\begin{abstract}
Colon cancer is the third most common cancer worldwide. Conventional therapies have shown moderate efficacy with severe adverse effects, therefore there is an urgent need for safer alternatives. In this study, Cistanche tubulosa, local name Thanoon, was considered as a potential phytotherapeutic strategy because of its known high therapeutic potential in traditional medicine and wide abundance in the Middle East region. Bioactive compounds were extracted from powdered Cistanche tubulosa and tested for their anticancer properties against four colon cancer cell lines including two derived from primary tumor (CaCo2 and HCT116) and two derived from metastatic site (LoVo and SW620). Effect of Cistanche tubulosa on induction of apoptosis and cellular redox homeostasis were also investigated. Cistanche tubulosa exhibited a concentration and timedependent inhibition of proliferation of all tested cancer cell lines by more than $60 \%$ upon 72 hours treatment with $1 \mathrm{mg} / \mathrm{mL}$ of crude extract. Inhibition of proliferation was marked by induction of apoptosis, intracellular reactive oxygen species production and mitochondrial superoxides. This data suggest that Cistanche tubulosa is a promising candidate for additive anti-colon cancer therapy. This is the first study showing anticancer bioactivity of Cistanche tubulosa against colon cancer cells.
\end{abstract}

\section{INTRODUCTION}

Colorectal cancer is one of the most common cancers worldwide (Brenner et al., 2014), and its incidence is constantly increasing with an estimated 2.4 million cases in 2035 , due to modern diet and lifestyle, along with reduced physical activity. Current efforts are not sufficient to combat the present epidemic of colorectal cancer and therefore novel approaches are needed for effective prevention and treatment including changes in life style in combination with safer alternative interventions such as

\footnotetext{
* Corresponding Author

Morana Jaganjac, PhD, Senior Scientist, Toxicology and Multipurpose

Labs, Anti Doping Lab Qatar, Sport City Road, Doha, Qatar.

Phone: +97444132846; Fax: +97444132997
}

Phytotherapeutics (Weidner et al., 2015). Phytotherapy, the use of medicinal plants to treat diseases, has been an inevitable part of ancient human history. Medicinal plants have long been utilized as alternative treatment sources for cancers, representing more than sixty percent of anticancer agents used in conventional medicine (Balunas and Kinghorn, 2005; Saibu et al., 2015).Some of the best known examples include extracts from Catharanthus roseus $\mathrm{G}$. Don. (Apocynaceae), Taxus baccata L. (Taxaceae) and Camptotheca acuminate Decne (Nyssaceae) (Cragg and Newman, 2005; da Rocha et al., 2001). Several herbal extracts and phytochemicals were shown to exert antitumor effects in colorectal cancer attributed to the induction of reactive oxygen species (ROS) production and associated apoptosis of cancer cells as the case of extracts from Melissa officinalis (Weidner et al., 2015). 
Among phytotherapeutic candidates, Cistanche tubulosa, an Orobanchaceae parasitic desert plant (Jiang et al., 2009) that is widely distributed in arid and semi-arid regions of Africa, Asia and the Mediterranean region, has been shown to have valuable medicinal properties. Cistanche tubulosa has been extensively used in traditional medicine, and suggested to have curative effects in kidney deficiency, morbid leucorrhea, metrorrhagia, female infertility, and senile constipation (Jiang et al., 2009).In addition to its traditional medicinal uses, important medicinal properties of Cistanche Tubulosa have been intensively studied during the last decade including vasorelaxant (Yoshikawa et al., 2006), hepatoprotective (Morikawa et al, 2010), anti-hyperglycemic and hypolipidemic effects (Xiong et al., 2013). Cistanche Tubulosa was also suggested as a potent enhancer of the immune system, promoter of bone formation, and an anti-aging and anti-fatigue agent (Xu et al., 2014). Furthermore, the Cistanche tubulosa extract has been shown to block amyloid deposition in Alzheimer's disease model (Wu et al., 2015). Despite its various therapeutic uses, the effect of Cistanche Tubulosa as a potential anticancer agent has not been studied yet.

In the present work we have investigated the anticancer effect of Cistanche Tubulosa on two primary and two metastatic colon cancer cell lines and the potential mechanisms underlying this effect.

\section{MATERIALS AND METHODS}

\section{Collection and preparation of plant extract}

The samples of Cistanche tubulosa were collected from desert area in Qatar during 2014 and the authenticity of the plant was confirmed by herbatologist. Voucher samples are archived in the Toxicology and Multipurpose Department at ADLQ. The sundried plant samples were grinded with Retsch Knife Mill Grindomix GM300 into fine powder. Twenty grams of powder were extracted with $200 \mathrm{~mL}$ ultrapure water over night at $37{ }^{\circ} \mathrm{C}$ on a rotary shaker at $200 \mathrm{rpm}$. The crude Cistanche tubulosa extracts (CTE) were centrifuged for $30 \mathrm{~min}$ at $8000 \mathrm{rpm}$ to pellet nonsoluble compounds, supernatant collected and freeze dried using Labconco Freezone 6 plus Freeze dryer. The dried extract was reconstituted in Dulbecco's Modified Eagle's Medium (DMEM, SIGMA, Germany) to a concentration of $20 \mathrm{mg} / \mathrm{mL}$ and sterile filtered through 0.2 micron membrane filter.

\section{Cell lines and cell maintenance}

Human colon carcinoma cell lines CaCo2, SW620 and LoVo were obtained from Cell Lines Service (CLS, Eppelheim, Germany) while HCT 116 cell line was a kind gift from the Biological and Environmental Sciences, Department at Qatar University. $\mathrm{CaCo} 2$ and HCT11 were derived from the primary site of colon carcinoma while SW620 and LoVo derived from metastatic site. SW620, HCT116 and LoVo cells were cultured and maintained in DME Medium while $\mathrm{CaCo} 2$ cells were maintained in Eagle's Minimum Essential Medium (EMEM, SIGMA, Germany). The cells were grown as monolayer cultured at $37^{\circ} \mathrm{C}$ in respective medium supplemented with $10 \%$ fetal bovine serum (FBS, SIGMA, Germany) and 1\% Penicillin/Streptomycin (SIGMA, Germany) in a humidified atmosphere containing $5 \%$ carbon dioxide.

\section{Cell viability assay}

The 3-[4,5-dimethylthiazol-2-yl]-2,5-diphenyltetrazolium (MTT) assay was used to evaluate the cytotoxic activity of CTE similarly as described earlier (Jaganjac et al., 2010). The seeding density of CaCo2, HCT116, SW620 and LoVo cells cultured in 96-well plates was $10^{4}$ cells per well. Cells were plated in respective medium supplemented with $10 \%$ FBS 24 hours prior to treatment. After 24 hours, the medium was removed and cells were treated with $0,0.25,0.5,1$ and $2 \mathrm{mg} / \mathrm{mL}$ of CTE for 24,48 and 72 hours at $37^{\circ} \mathrm{C}$ in a humidified atmosphere containing $5 \% \mathrm{CO}_{2}$. Upon CTE treatment, the medium was removed and $40 \mu \mathrm{L}$ of MTT solution $(0.5 \mathrm{mg} / \mathrm{mL})$ added to each well.

After $3 \mathrm{~h}$ of incubation, MTT solution was removed, the formazan product dissolved in dimethylsulfoxide (DMSO, SIGMA, Germany), and the absorbance measured at $590 \mathrm{~nm}$ with microplate reader (Infinite 200 PRO NanoQuant, Tecan Trading AG, Switzerland).

\section{Apoptosis assay}

Apoptosis in CaCo2, HCT116, SW620 and LoVo cells was detected using PE Annexin V Apoptosis Detection Kit I with 7-Amino-Actinomycin D (7-AAD) as a vital dye (Becton Dickinson International, Belgium) according to the manufacturer's instruction. Briefly, cells were seeded in 24-well plates at a density of $5 \times 10^{4}$ cells/well in a respective media supplemented with $10 \%$ FBS for $24 \mathrm{~h}$ prior to the addition of CTE $(0,0.5$ or $1 \mathrm{mg} / \mathrm{mL})$. Following 24 CTE incubation, cells were harvested, washed twice with cold phosphate-buffered saline and stained with Phycoerythrin (PE) Annexin V and 7-AAD for $15 \mathrm{~min}$ at room temperature in the dark. Stained cells were analyzed within 1 hour by flow cytometry using FACS. Aria III flow cytometer and FACSDiva software (Becton Dickinson) at a low flow rate with a minimum of $10^{4}$ cells. Treatment of cells for 4 hours with $6 \mu \mathrm{M}$ camptothecin (SIGMA) was used as a positive control for the assay.

\section{Intracellular ROS production}

Intracellular ROS production was examined using 2,7dichlorodihydrofluorescein diacetate (DCFH-DA, SIGMA, Germany). DCFH-DA is a nonfluorescent probe, which is oxidized with intracellular ROS to the fluorescent compound 2,7dichlorofluorescein (DCF) (Poljak-Blazi et al., 2011). The DCFHDA assay was performed similarly as we have described before (Cindric et al, 2013; Poljak-Blazi et al., 2011). Briefly, the seeding density of CaCo2, HCT116, SW620 and LoVo cells cultured in 96-well black plates was $10^{4}$ cells per well. Cells were plated in respective medium supplemented with $10 \%$ FBS for 24 hours. Prior to treatment cells were incubated with $10 \mu \mathrm{M}$ DCFH-DA at $37^{\circ} \mathrm{C}$ for $30 \mathrm{~min}$ in $5 \% \mathrm{CO}_{2} / 95 \%$ air. Cells were then washed and 
treated with $0,0.5$ and $1 \mathrm{mg} / \mathrm{mL}$ of CTE in the medium w/o phenol red. The intracellular ROS formation was monitored continuously throughout 25 hours at $37^{\circ} \mathrm{C}$ and $5 \% \mathrm{CO}_{2}$ using microplate reader with top fluorescence and gas control module (Infinite 200 PRO, Tecan Trading AG, Switzerland). The fluorescence intensity was measured with an excitation wavelength of $500 \mathrm{~nm}$ and emission detection at $529 \mathrm{~nm}$. The arbitrary units, relative fluorescence units (RFU), were based directly on fluorescence intensity.

\section{Mitochondrial superoxide generation}

The ability of CTE to induce superoxide generation by mitochondria was estimated using cell-permeable, mitochondria targeted MitoSOX Red probe (Life Technologies) and Hoechst 33342 for nuclear staining (Life Technologies). CaCo2, HCT116, SW620 and LoVo cells were seeded in 96-well plates at a density $10^{4}$ cells per well in a respective medium supplemented with $10 \%$ FBS for 24 hours. Cells were then loaded with $4 \mu \mathrm{M}$ of MitoSOX and $2 \mu \mathrm{M}$ of Hoechst 33342 for $20 \mathrm{~min}$, excess dye washed and wells treated with $0,0.5$ and $1 \mathrm{mg} / \mathrm{mL}$ of CTE for 24 hours at $37^{\circ} \mathrm{C}$ and $5 \% \mathrm{CO}_{2}$. The fluorescence intensity was measured with an excitation wavelength of $510 \mathrm{~nm}$ and emission detection at $580 \mathrm{~nm}$ for MitoSOX and an excitation wavelength of $350 \mathrm{~nm}$ and emission detection at $461 \mathrm{~nm}$ for Hoechst 33342 using microplate reader with top fluorescence (Infinite 200 PRO, Tecan Trading AG, Switzerland)

\section{Statistical analysis}

Descriptive statistics were shown as the mean +/- SD.

The significance of differences between groups was assessed using the Student t-test and Chi-square test. When more than two groups were compared, we used one sided ANOVA with appropriate post hoc testing. The SPSS 11.01 for Mircosoft Windows were used. Differences with $\mathrm{P}$ less than 0.05 were considered statistically significant.

\section{RESULTS}

The effect of CTE on proliferation of human colon cancer cell lines is shown in Figure 1. All CTE concentrations

A

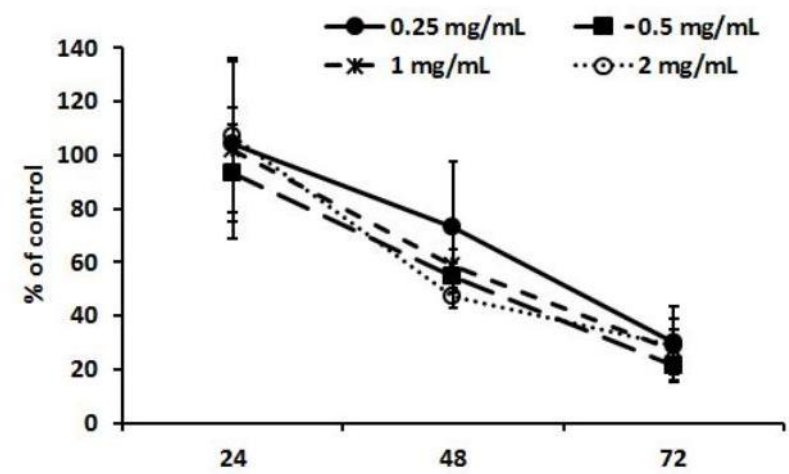

tested showed a strong inhibitory effect on $\mathrm{CaCo} 2$ cell line in a concentration and time dependent manner (Figure 1A). Seventy two hours of CTE treatment inhibited the growth of $\mathrm{CaCo} 2$ cells by more than $60 \%$ compared to control ( $p<0.05$ for all concentrations). Significant impact of CTE treatment on HCT116 cell growth was also detected at all-time points at the two highest concentrations $(1 \mathrm{mg} / \mathrm{mL}$ and $2 \mathrm{mg} / \mathrm{mL})$, reaching greater than $70 \%$ reduction at latter concentration (Figure $1 \mathrm{~B}, \mathrm{p}<0.05$ ). Although the two lower CTE concentrations $(0.25$ and $0.5 \mathrm{mg} / \mathrm{mL})$ significantly reduced HCT116 growth of cells after 24 hours $(\mathrm{p}<0.05)$, they had no significant effect following 72 hours treatment compared to control $(p>0.05)$. Time and concentration dependent inhibition of proliferation with CTE was further confirmed in LoVo cells by more than $60 \%$ at highest concentration $(\mathrm{p}<0.05)$ (Figure 1C), and all four CTE concentrations tested reduced the growth of SW620 cells after 48 hours (Figure 1D, p < 0.05 for all). After 72 hours of treatment the same effect was observed only for the two highest concentrations ( $\mathrm{p}<0.05$ ) while 0.25 and $0.5 \mathrm{mg} / \mathrm{mL}$ concentrations showed no significant effect compared to control ( $p>0.05)$. The impact of 0.5 and $1 \mathrm{mg} / \mathrm{mL}$ CTE treatment on induction of apoptosis was further tested in all four cell lines (Figure 2). Increased number of cells in early apoptosis was detected in HCT116 and LoVo following 24 hours treatment with $0.5 \mathrm{mg} / \mathrm{mL}(\mathrm{p}<0.05$, Figure $2 \mathrm{~B}$ and $2 \mathrm{C})$ and in all cell lines at $1 \mathrm{mg} / \mathrm{mL}(\mathrm{p}<0.05)$. Significant increase in necrotic or late apoptotic cell number was further observed in $\mathrm{CaCo} 2$ and SW620 cell lines ( $<<0.05$, Figure $2 \mathrm{~A}$ and 2D). Ability of CTE to induce intracellular ROS production is demonstrated in Figure 3. Three hours following CTE treatment there was a strong increase in intracellular ROS production in all cell lines $(\mathrm{p}<0.05)$. The intracellular ROS production increased progressively throughout the 25 hours treatment in a time and concentration dependent manner. Furthermore, staining of cells with mitochondria targeted probe revealed a strong impact of CTE on mitochondrial superoxide production in a concentration dependent manner (Figure 4). Highest increase in superoxide production by mitochondria was observed in HCT116 (69\%, Figure 4B) and LoVo cells (82\%, Figure 4C) following 24 hours treatment with 1 $\mathrm{mg} / \mathrm{mL}$ of CTE.
B

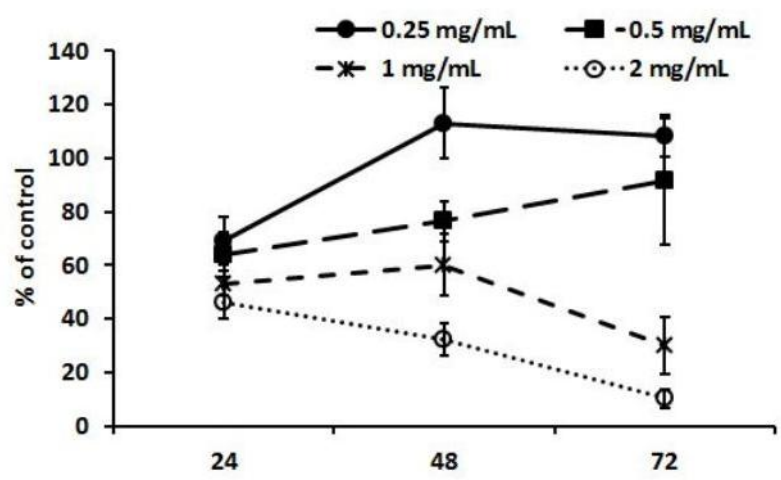


C

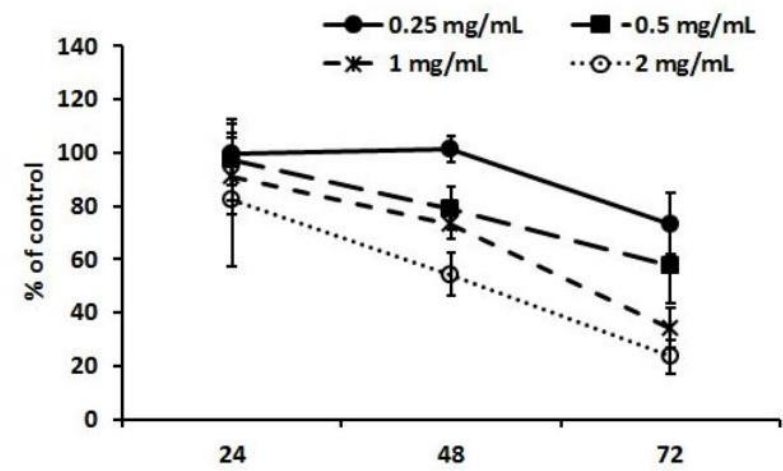

D

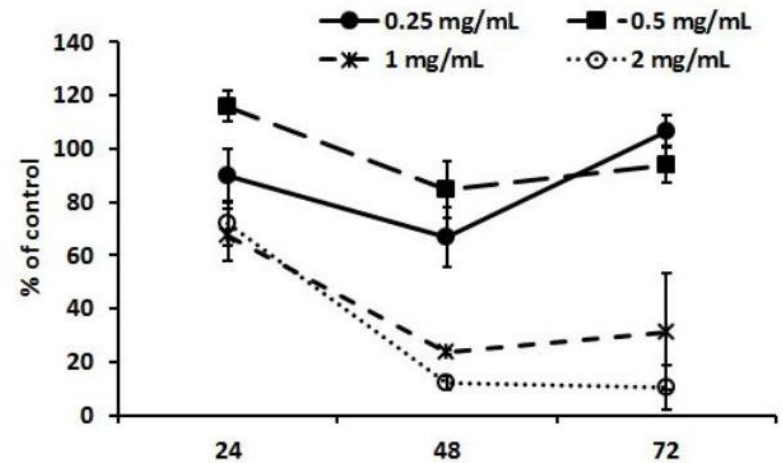

Fig. 1: Effect of Cistanche tubulosa on the viability of colon cancer cell lines. Cell viability measured by MTT assay of (A) CaCo2, (B) HCT116, (C) LoVo and

(D) SW620 cells are presented as percentage of control untreated colon cancer cell line. Mean values $( \pm \mathrm{SD})$ for 5-replicates of representative experiment is given: $\left.{ }^{*}\right)$ significance $\mathrm{p}<0.05$ in comparison to control untreated respective cells.

A

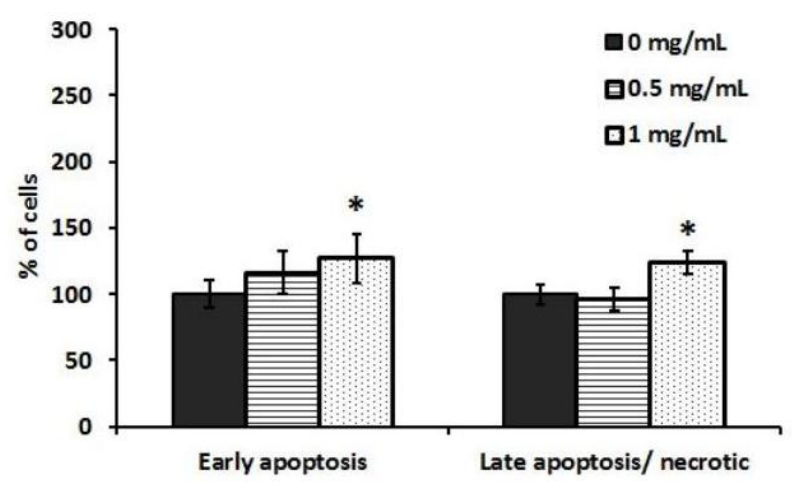

C

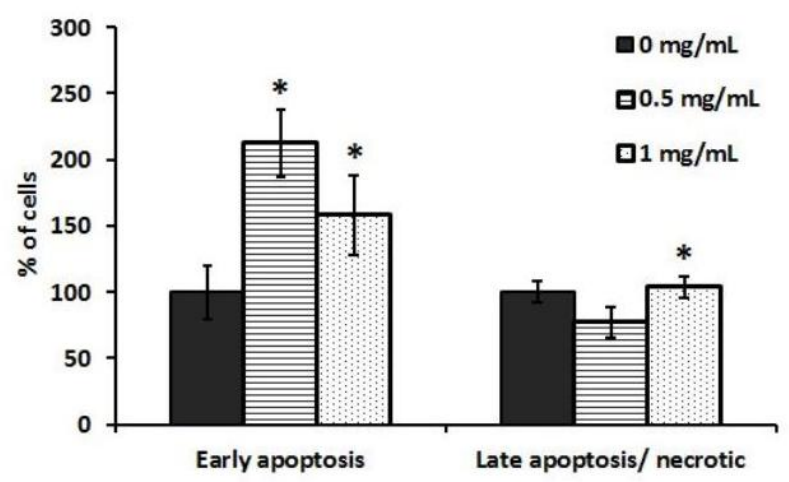

B

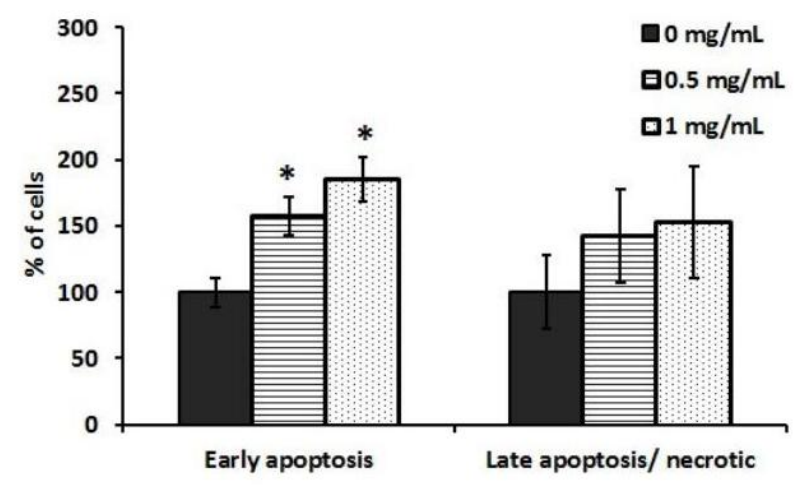

D

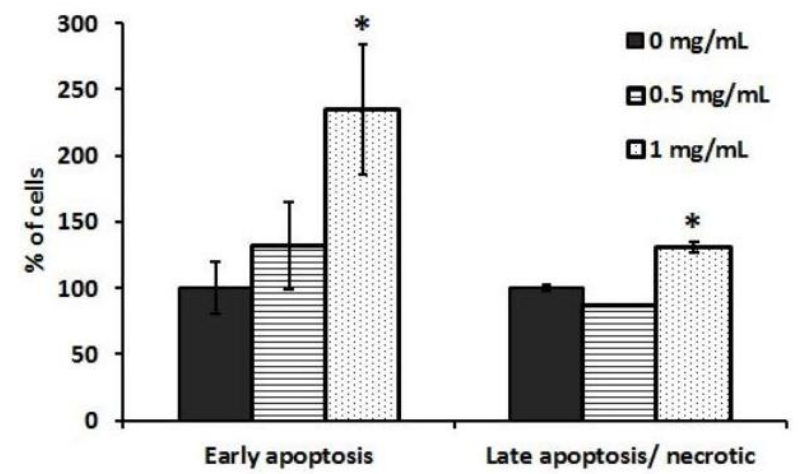

Fig. 2: Cistanche tubulosa water extract induces apoptosis in human colon cancer cells. Annexin-V-FITC flow cytometry analyses of (A) CaCo2, (B) HCT116, (C) LoVo and (D) SW620 cells are presented as percentage of control untreated colon cancer cell line. Mean values ( \pm SD) for 3-replicates of representative experiment is given: $(*)$ significance $\mathrm{p}<0.05$ in comparison to control untreated respective cells. 
A

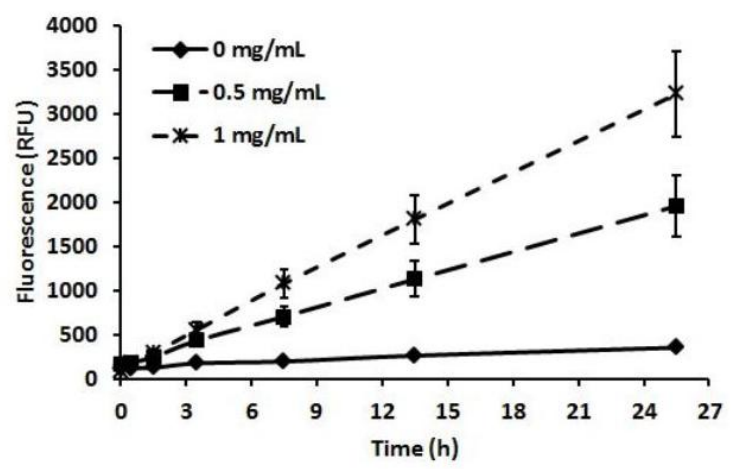

C

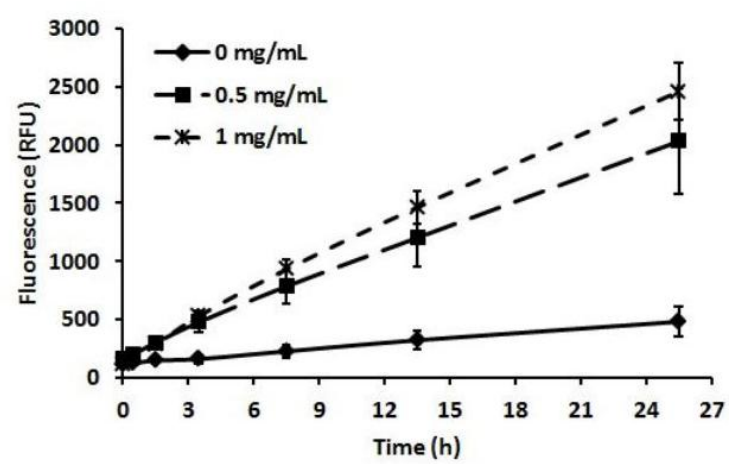

B

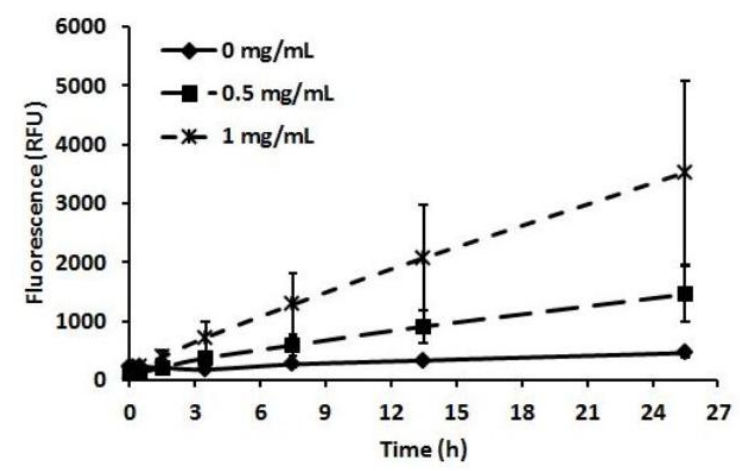

D

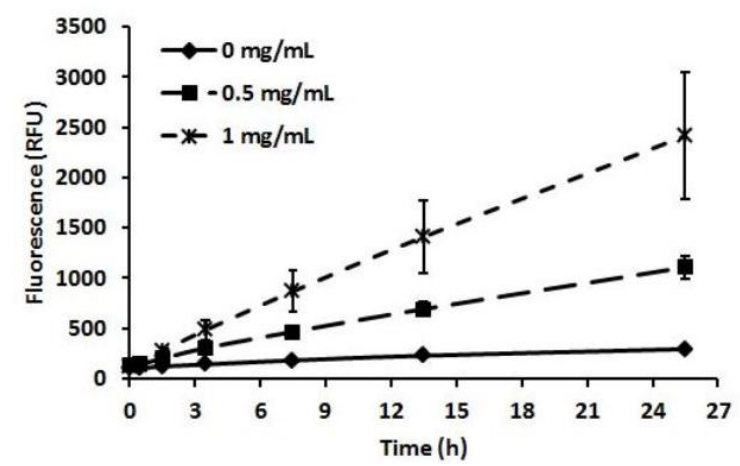

Fig. 3: Cistanche tubulosa water extract induces intracellular ROS production in a time- and dose-dependent. ROS production measured by DCFH-DA assay in (A) $\mathrm{CaCo}$, (B) HCT116, (C) LoVo and (D) SW620 cells is presented as mean RFU values ( \pm SD) for the respective 5-replicates of representative experiment. (*) Significance $\mathrm{p}<0.05$ in comparison to control untreated colon cancer cells.

A

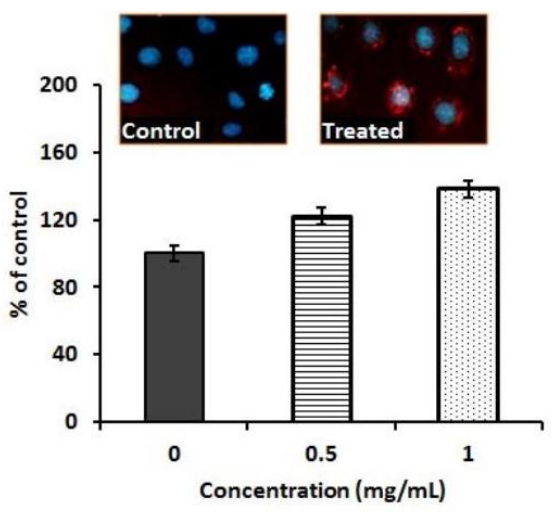

C

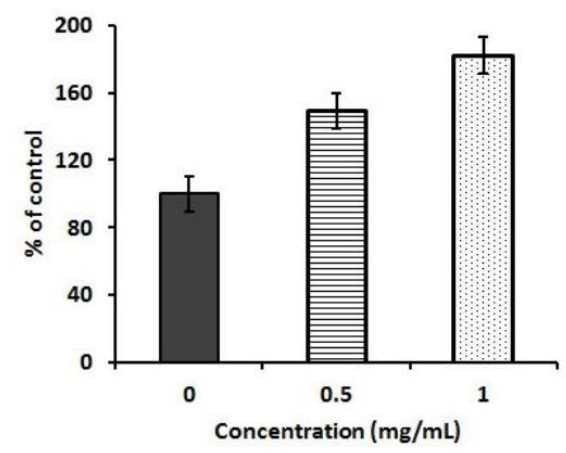

B

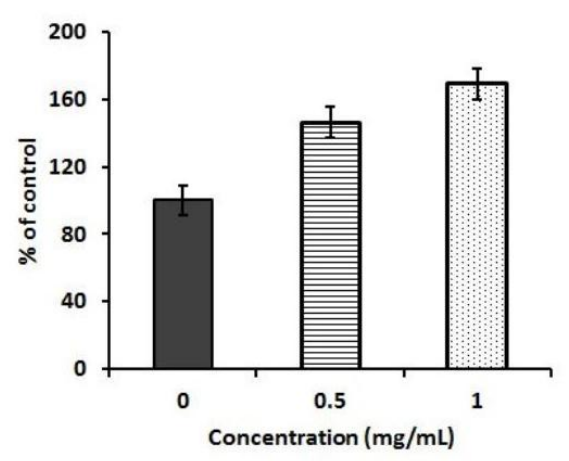

D

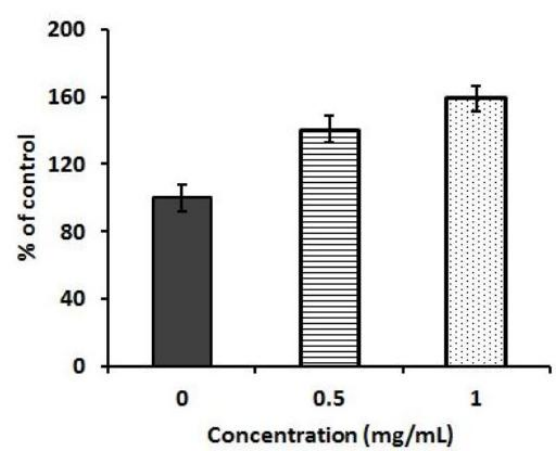

Fig. 4: Cistanche tubulosa water extract induces mitochondrial superoxide production in human colon cancer cells. Fluorescence intensity of mitochondria targeted MitoSOX Red probe in A) CaCo2, (B) HCT116, (C) LoVo and (D) SW620 cells are presented as percentage of control untreated colon cancer cell line. Mean values $( \pm \mathrm{SD})$ for 5 -replicates of representative experiment is given: $(*)$ significance $\mathrm{p}<0.05$ in comparison to control untreated colon cancer cells. 


\section{DISCUSSION}

Although earlier studies have reported numerous medicinal properties of Cistanche tubulosa, this is the first report of its anti proliferative effect in malignant cells. Cistanche tubulosa bioactive compounds extracted with water, a highly polar solvent, showed strong anticancer bioactivity. We have previously compared the efficiency of Cistanche tubulosa solubilized in water against other solvents such as methanol and ethyl acetate, but water extracts exhibited the most promising anticancer activities (data not shown).

We have demonstrated the ability of CTE at $1 \mathrm{mg} / \mathrm{mL}$ and $2 \mathrm{mg} / \mathrm{mL}$ to inhibit $60 \%$ of the growth of both primary and metastatic colon cancer cell lines, revealing a potential important role of Cistanche tubulosa as a colon cancer treatment. Compared to normal cells, cancer cells are generally characterized by a disturbance in redox homeostasis and a common strategy of current anticancer therapies is to increase cellular oxidative stress (Yang et al, 2013). Although physiologically low levels of ROS have important role as signaling molecules, the excessive ROS production can contribute to cancer instability and malignancy (Liou and Storz, 2010). Paradoxically, this imbalance in cellular redox homeostasis renders cancer cells more vulnerable to ROSinduced cell death (Jaganjac et al., 2008; Nogueira and Hay, 2013). The anti-proliferative effect of CTE reported in this study can be mediated by various extra- and intracellular mechanisms of known and unknown compounds within the extract, targeting multiple pathways that play essential roles in apoptosis. In order to differentiate between different modes of cell death, we investigated the potential mechanism responsible for the observed CTE-induced cytotoxicity.

Our data indicate that CTE increases intracellular ROS production and consequently ROS-induced cell death. Redox state of the cell also plays a crucial role in regulating apoptosis and mitochondrial electron transport chain is one of the major sites of cellular ROS generation (Trachootham et al., 2008). Furthermore, the intracellular ROS could cause cellular apoptosis via both mitochondria-dependent and independent pathways (Sinha et al., 2013). Indeed, our data also indicate that CTE induced phosphatidylserine externalization, a common effect in apoptosis, in both primary and metastatic cancer cell lines, suggesting that the mechanism of CTE induced death is mediated by apoptosis rather than necrosis. The activation of apoptosis in cancer cells is a corrective strategy and many anticancer drugs may exert apoptotic effects in cancer cells.

Compounds or extracts with pro-apoptotic activities in cancer cells are therefore potentially useful in anticancer drug research (Wong, 2011). In order to determine whether CTE induced pro-apoptotic effect is mediated by mitochondria-induced ROS mechanism, we measured superoxide production using mitochondria targeted fluorescent probe in CTE treated cells.Our data clearly shows that CTE stimulates mitochondrial superoxide production suggesting that Cistanche tubulosa anticancer activity is at least in part mediated through mitochondria induced ROS mechanism.

\section{CONCLUSIONS}

In conclusion, our data suggests that the water extract of desert plant Cistanche tubulosa may represent a promising candidate for anticancer approach in combination with other conventional therapies for prevention and treatment of colon cancer. We also demonstrate that the toxicity of the plant extract against cancer cells is mediated by increased intracellular ROS production and, at least in part, by mitochondrial-dependent apoptosis. Further studies are ongoing to isolate and characterize the individual biologically active constituents responsible for anticancer activity.

More research is needed to evaluate the potential use of this extract as an effective chemopreventive agent and to understand the mechanisms of action on colon cancer cells at the molecular level. Further preclinical and clinical studies are also needed to confirm the observed beneficial health effects of Cistanche tubulosa for cancer prevention.

\section{ACKNOWLEDGEMENTS}

Financial support and sponsorship: This study was supported by the Anti Doping Lab Qatar.

Conflict of Interests: The authors' declare no conflict of interest.

\section{REFERENCES}

Balunas MJ, Kinghorn, AD. Drug discovery from medicinal plants. Life Sci. 2005;78:431-41.

Brenner H, Kloor M, Pox CP. Colorectal cancer. Lancet 2014;383,1490-502.

Cindric M, Cipak A, Zapletal E, Jaganjac M, Milkovic L, Waeg G, Stolc S, Zarkovic N, Suzana Borovic S. Stobadine attenuates impairment of an intestinal barrier model caused by 4-hydroxynonenal. Toxicol In Vitro. 2013;27:426-32.

Cragg GM, Newman DJ. Plants as a source of anticancer agents.JEthnopharmacol. 2005;100:72-9.

da Rocha AB, Lopes RM, Schwartsmann G. Natural products in anticancer therapy. CurrOpinPharmacol. 2001;1:364-9.

Jaganjac M, Matijevic T, Cindric M, Cipak A, Mrakovcic L, Gubisch W, Zarkovic N. Induction of CMV-1 promoter by 4-hydroxy-2nonenal in human embryonic kidney cells. Acta Biochim Pol. 2010;57:179-83.

Jaganjac M, Poljak-Blazi M, Zarkovic K, Schaur RJ, Zarkovic $\mathrm{N}$. The involvement of granulocytes in spontaneous regression of Walker 256 carcinoma. Cancer Lett. 2008;260:180-6.

JiangY, Tu PF. Analysis of chemical constituents in Cistanche species. J Chromatogr A. 2009;1216:1970-9.

Liou GY, Storz P. Reactive oxygen species in cancer. Free Radic Res. 2010;44:479-96.

Morikawa T, Pan Y, Ninomiya K, Imura K, Matsuda $\mathrm{H}$, Yoshikawa M, Yuan D, Muraoka O. Acylated phenylethanoid oligoglycosides with hepatoprotective activity from the desert plant Cistanche tubulosa. Bioorg Med Chem. 2010;18:1882-90.

Nogueira V, Hay N. Molecular pathways: reactive oxygen species homeostasis in cancer cells and implications for cancer therapy. Clin Cancer Res. 2013;19:4309-14. 
Poljak-Blazi M, Jaganjac M, Sabol I, Mihaljevic B, Matovina M, Grce M. Effect of ferric ions on reactive oxygen species formation, cervical cancer cell lines growth and E6/E7 oncogene expression. ToxicolIn Vitro. 2011;25:160-6.

Saibu GM, Katerere DR, Rees DJG, Meyer M. In vitro cytotoxic and pro-apoptotic effects of water extracts of Tulbaghia violacea leaves and bulbs. J Ethnopharmacol. 2015;164:203-9.

Sinha K, Das J, Pal PB, Sil PC. Oxidative stress: the mitochondria-dependent and mitochondria-independent pathways of apoptosis. Arch Toxicol.2013; 87:1157-80.

Trachootham D, Lu W, Ogasawara MA, Nilsa RD, Huang P. Redox regulation of cell survival. Antioxid Redox Signal. 2008;10:134374.

Weidner C, Rousseau M, Plauth A, Wowro SJ, Fischer C, Abdel-Aziz H, Sauer S. Melissa officinalis extract induces apoptosis and inhibits proliferation in colon cancer cells through formation of reactive oxygen species. Phytomedicine. 2015;22:262-70.

Wong RS. Apoptosis in cancer: from pathogenesis to treatment. J ExpClin Cancer Res. 2011;30:87.

Wu S-H, Chou F-P, Chyau C-C, Chen J-H, Tu S-F, Lin H-H. Anti-cancerous effects of Wasabia japonica extract in Hep3B liver cancer cells via ROS accumulation, DNA damage and p73-mediated apoptosis. J Funct Foods. 2015;14:445-55.

Xiong W-T, Gu L, Wang C, Sun H-X, Liu X. Antihyperglycemic and hypolipidemic effects of Cistanche tubulosa in type 2 diabetic db/db mice. J Ethnopharmacol. 2013;150:935-45.
Xu R, Sun S, Zhu W, Xu C, Liu Y, Shen L, Shi Y, Chen J. Multi-step infrared macro-fingerprint features of ethanol extracts from different Cistanche species in China combined with HPLC fingerprint. J Mol Struct. 2014;1069:236-244.

Yang Y, Karakhanova S, Werner J, Bazhin AV. Reactive oxygen species in cancer biology and anticancer therapy. Curr Med Chem. 2013;20:3677-92.

Yoshikawa M, Matsuda H, Morikawa T, Xie H, Nakamura S, Muraoka O. Phenylethanoid oligoglycosides and acylated oligosugars with vasorelaxant activity from Cistanche tubulosa. Bioorg Med Chem. 2006; 14:7468-75.

\section{How to cite this article:}

Al-Menhali AS, Jameela SA, Latiff AA, Elrayess MA, Alsayrafi $\mathrm{M}$, Jaganjac M. Cistanche tubulosa induces reactive oxygen species-mediated apoptosis of primary and metastatic human colon cancer cells. J App Pharm Sci, 2017; 7 (05): 039-045. 